T. Kasraoui, et al., Int. J. Comp. Meth. and Exp. Meas., Vol. 5, No. 1 (2017) 44-54

\title{
MODELING OF UNSTEADY HEAT TRANSFER BY IMPACT BETWEEN GAS PARTICLES AND A COLD WALL IN A SPHERICAL COMBUSTION VESSEL
}

\author{
T. KASRAOUI, ${ }^{1,2}$ K. JOULAIN, ${ }^{2}$ R. BERTOSSI, ${ }^{1}$ M. BELLENOUE, ${ }^{3}$ B. BOUST $^{3}$ \& J. SOTTON ${ }^{3}$ \\ ${ }^{1}$ DRII, IPSA, Ivry-sur-Seine, France. \\ ${ }^{2}$ Institut Pprime, département Fluide, Thermique et Combustion, ENSIP, Poitiers, France. \\ ${ }^{3}$ Institut Pprime, département Fluide, Thermique et Combustion, ISAE-ENSMA, \\ Futuroscope Chasseneuil, France.
}

\begin{abstract}
To evaluate the wall heat losses in combustion vessel, an alternative to existing macroscopic models of heat transfer is suggested. This study aims to provide a physical approach for wall heat transfer based on kinetic theory of gases to describe the conduction phenomena between gas particles and the cold wall in short scales. The model mentioned is implemented in a code simulating combustion in a constant volume spherical chamber.

Keywords: ballistic regime, combustion, kinetic theory of gases, unsteady heat transfer.
\end{abstract}

\section{INTRODUCTION}

The study of wall heat losses in internal combustion engines is of crucial importance as they affect engine performances. However, the presence of high temperatures and high pressures in combustion chambers creates a competition between the performance improvement and the heat losses increasing. Estimating the heat losses is, then, required to help the process of engine design and to set the boundary conditions. For this purpose, the evaluation of wall heat losses must be based on a better modeling of involved physical phenomena, as the amount of the wall heat losses in a combustion chamber represents about one third of the chemical energy of combustion in conventional engines.

Numerical simulations of wall heat losses in a combustion chamber must take into account the flame-wall interaction and thus, the reactive aspect of the flow, which is important for the understanding of near wall combustion [1]. In this work, a spherical combustion chamber at constant volume for a stoichiometric methane-air mixture is chosen. These conditions are representative of piston engine conditions where both thermal and aerodynamic behaviors of the flame-wall interaction are described in a simplified manner. Our approach consists in the modeling of the parietal flow by introducing a ballistic heat transfer model, which represents the novelty of this work.

\section{STATE OF THE ART OF HEAT LOSS MODELING}

2.1 Theoretical aspects of flame-wall interaction and burnt gases-wall interaction in a closed combustion vessel at constant volume

Modeling of the wall heat losses requires distinction between two phenomena: the flame-wall interaction and the burnt gases wall interaction. The flame-wall interaction involves several factors that depend on the nature of the flow regime. For the combustion in a constant volume chamber, the flame stops propagating at a distance from the wall, $\delta_{q}$. This extinction, called the quenching phenomenon, is due to the presence of parietal thermal losses coupled or not 
to the stretching flame front or the radical de-excitation by the wall. It depends on many parameters such as the surface state and the burning medium. Among them, we can include the Peclet number $P_{e q}$ relative to quenching. It represents the ratio between the quenching distance and the thickness of unstretched laminar flame $\delta_{l}$ defined as: $\delta_{l}=\lambda / \rho_{u} C_{p} S_{I}$, where $\lambda$ is the thermal conductivity of fresh gas $\left[\mathrm{W} \cdot \mathrm{m}^{-1} \cdot \mathrm{K}^{-1}\right], \rho_{u}$ is the fresh gases density $\left[\mathrm{kg} \cdot \mathrm{m}^{-3}\right]$, $C_{p}$ is the thermal capacitance of fresh gases $\left[\mathrm{J}^{\mathrm{kg}} \mathrm{kg}^{-1} \cdot \mathrm{K}^{-1}\right]$ and $S_{l}$ is the non-stretched laminar flame velocity $\left[\mathrm{m} . \mathrm{s}^{-1}\right]$. Huang et al. [2] found that the Peclet number in laminar flow toward a wall, maintained at low temperature $\left(25^{\circ} \mathrm{C}\right)$, is about 3-4 for a head-on quenching where the flow is perpendicular to the wall. Another important parameter is represented by the dimensionless heat flux $\varphi$ defined as following:

$$
\varphi=\frac{Q_{w}}{Q_{l}}
$$

where $Q_{w}$ is the parietal heat flow and $Q_{l}$ is the heat power of an unstretched laminar flame defined as: $Q_{l}=\rho_{u} S_{l} Y_{\text {fuel }} \Delta H$, where $\mathrm{Y}_{\text {fuel }}$ is the fuel mass fraction and $\Delta H$ is the reaction heat

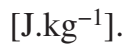

\subsection{Previous works of parietal heat flux modeling in combustion chambers}

In the past decade, many theoretical and experimental works studied the quenching phenomenon in different geometric configurations. For example, Popp and Baum [3] analyzed the wall heat flux during the head-on quenching of a laminar methane-air flame.

\subsubsection{The quenching distance modeling}

Potter and Berlad [4] proposed a formulation of $\delta_{q}$ based on the reaction rate and compared the results to experiments. This model predicts the quenching distance according to the following expression:

$$
\delta_{q}^{2}=k_{2} \cdot\left(1-\frac{Q_{w}}{Q_{l}}\right) \frac{\lambda X_{f u e l}}{C_{p} \cdot w}
$$

with $k_{2}$ is an empirical coefficient, $X_{\text {fuel }}$ is the molar fraction of the fuel in fresh gases and $w$ is the reaction rate. This modeling requires the knowledge of the preheated zone temperature and an empirical coefficient whose interpretation remains difficult.

Boust et al. [5] established another formulation of the quenching distance based on the energy balance at the wall. It is an analytical relation between the Peclet number at the quenching moment and the non-dimensional heat flux, eqn (3):

$$
\varphi=\frac{1}{P e+1} \Leftrightarrow P_{\mathrm{e}}=\frac{1-\varphi}{\varphi}
$$

This model remains available only in low-fresh gas compression.

\subsubsection{The parietal heat flux modeling}

In the literature, many theoretical studies have estimated heat losses in combustion chambers. Most of the corresponding formulations considered only the conduction mode in a parietal 
thermal boundary layer. Heat transfer models usually provide either the wall heat flux, $Q_{w}$, or a heat transfer coefficient, h, which are related by Newton's law:

$$
Q_{w}=h \cdot\left(T_{g}-T_{w}\right)
$$

with $\mathrm{T}_{\mathrm{g}}$ is the hot gas temperature and $\mathrm{T}_{\mathrm{w}}$ is the wall temperature.

Another model established by $[6,7]$ predicts the stationary heat flux. It is valid only at low pressures, less than $1 \mathrm{~atm}[8]$ :

$$
Q_{w}=\lambda\left(T_{g}-T_{w}\right) / \delta_{q}
$$

\subsubsection{Empirical correlations}

Numerous empirical models have been established in the past to compute wall heat losses. One of them is based on the assumption of convective heat transfer of Nusselt [9]. This formulation quantifies the heat transfer coefficient from experiments of laminar combustion in a spherical vessel which takes into account the mean gas temperature $T_{g}$, the pressure $\mathrm{P}(\mathrm{MPa})$ and the mean piston speed $C_{m}$. The heat transfer coefficient is expressed in two different cases as follows:

Spherical bomb: $h=1.15 \sqrt[3]{P^{2} T_{g}}$

Engine cylinders: $h=1.15\left(1+1.24 C_{m}\right) \sqrt[3]{P^{2} T_{g}}$

This model underestimates heat losses and involves the mean gas temperature, which does not account for the gap on local temperature due to combustion.

In order to take into account the global aerodynamic phenomena, other formulations have been proposed. Among them, Woschni [10] used the forced convection assumption and involved a characteristic velocity, w:

$$
\begin{gathered}
h=130 D^{-0.2} P^{0.8} T^{-0.53} w^{0.8} \\
w=2.28 C_{m}+0.00324 \frac{V_{c y l} T_{1}}{P_{1} V_{1}}\left(P-P_{0}\right)
\end{gathered}
$$

with $P$ (bar), $V_{c y l}$ is the cylinder volume, $P_{0}$ is the reference pressure the index 1 refers to the $\mathrm{BDC}$ conditions and $D$ is the cylinder bore.

Unlike most correlations that are based on convective heat transfer, most of them have major drawbacks and requires experimental data for each configuration.

\subsubsection{Laws of the wall}

In order to improve the heat loss modeling, local formulations of heat transfer have been developed. They are based on physical phenomena occurring near the wall. The model developed by Han et al. [11] is based on the kinematic boundary layer and estimates heat losses in stationary and compressible flows.

Other formulations, such as the model derived by Rivère and Mechkor [12], come from an atomic-scale interpretation of gas-wall collisions and take into account the presence of the thermal boundary layer near the wall. Wall heat flux is considered as the statistical result of collisions between gas molecules and the wall. The heat transfer coefficient is expressed as follows:

$$
h=\rho_{g} \sqrt{T_{g}} \sqrt{2 / \pi}\left(\frac{R}{M}\right)^{3 / 2}\left(\eta+\frac{\kappa}{\sqrt{T_{w}}}-\frac{\lambda}{T_{w}}\right)
$$


with $\rho_{g}$ is the gas density $\left(\mathrm{kg} \cdot \mathrm{m}^{-3}\right), M$ is the gas molar mass $\left(\mathrm{kg} \cdot \mathrm{mol}^{-1}\right), T_{g}$ is the fresh gas temperature and $T_{w}$ is the wall temperature. Empirical parameters such as $\lambda$ and $\kappa$ refer to the wall properties among which density, thermal conductivity and specific heat. $\eta$ depends on flow motion.

Built on a physical basis, this model accounts for wall heat losses by integrating the one-dimensional heat transfer equation in laminar combustion. Nevertheless, it still has some shortcomings as it requires empirical parameters.

State-of-the-art models still have major drawbacks, as none of them is universally applicable and do not reproduce experimental results with good accuracy. In this study, another model is, hence, used. Heat losses are then investigated following an original approach based on the kinetic theory of gases where collisions between gas particles in the ballistic regime play a major role.

\section{NUMERICAL SIMULATION: FLAME-WALL INTERACTION AND BURNT GAS-WALL INTERACTION}

In this part, we present a numerical study developed to simulate the thermodynamic aspects in a spherical combustion chamber at constant volume. Numerical results are then compared to experimental measurements of wall heat flux to validate the model.

\subsection{Experimental data of reference}

The experimental device consists in measuring the heat transfer flux in a fundamental shape of the combustion chamber. Data are provided by Boust [1] and experiments are carried in a constant volume spherical vessel as it represents local engine conditions where the volume remains nearly constant. The time evolution of pressure is recorded with a piezoelectric transducer and a heat flux gauge based on a thin film thermocouple (TFT) records the wall heat losses. The combustion chamber is operated with stoichiometric methane-air mixtures of initial pressure of $0.4 \mathrm{MPa}$ and initial temperature of $300 \mathrm{~K}$. The spherical vessel is made of steel of $82 \mathrm{~mm}$ of diameter.

\subsection{Expression of wall heat flux}

The parietal heat flux model used in this study is based on the kinetic theory of gases. It includes the concept of the distribution function, $f(r, v, t)$, which describes the particle velocities in idealized gases and gives the probability, per unit velocity, of the particle number, $d n(r, v, t)$, having at time $\mathrm{t}$, a position in the volume element $\mathrm{dr}^{3}$ and a speed centered in the volume element $\mathrm{dv}^{3}$. It uses also, the Boltzmann equation which describes the evolution of the distribution function [13].

Our model describes the thermal conduction at microscopic scales, in a regime where the distance considered are smaller than the molecules mean free path. We are therefore in a regime where there are no interactions between particles and where molecules carry ballistically heat from the gas to the wall. Note that consideration of the boundary conditions with the wall is essential. For non-thermodynamic local equilibrium systems, conductive heat flux in the ballistic regime calculated between two walls separated by a short distance (less than the mean free path), is described below:

$$
\boldsymbol{Q}_{\text {cond }}=\boldsymbol{h} * \Delta \boldsymbol{T}, \boldsymbol{h}=\frac{n T_{w}\left(2 k_{b}\right)^{3 / 2}}{2 \sqrt{\pi m T_{w}}}
$$


With $n$ is the gas density near the wall $\left(\mathrm{m}^{-3}\right), k_{b}$ is the Boltzmann constant and $m$ is the fresh gas molecule mass $(\mathrm{kg})$.

\subsection{Description of the numerical code}

The model mentioned is implemented in a FORTRAN code simulating combustion in a constant volume spherical chamber. Numerical simulations consist in modeling combustion phenomena by taking into account the resolution of the one-dimensional transient heat equation in the gas in spherical coordinates. As the laminar flame propagates from the center of the chamber, combustion is simulated by a thermal network, which is built with analogy with $(R, C)$ electric circuits. Therefore, flame propagation is approximated by the virtual combustion of discrete gas slices. Near the wall, the $\mathrm{N}-1$ slices of fresh gases are defined at equal volume and the thickness of the last slice is equal to the mean free path to apply the heat transfer model in ballistic regime.

Computations take into account a sequence of thermodynamic transformations: an isobaric combustion, heat losses by conduction and radiation and an isentropic re-compression of all fluid slices to obtain a constant volume combustion. As the flame approaches the wall, the combustion phase stops according to the quenching criterion. The cooling phase is then initiated and simulated by the same steps, except isobaric combustion.

\subsubsection{Isobaric combustion}

During combustion phase and at each time step, the unstretched laminar flame propagates in fresh gases at a constant speed defined by Metghalchi and Keck [14] as following:

$$
S_{u}(T, P)=S_{u 0}\left(\frac{T}{T_{0}}\right)^{\alpha}\left(\frac{P}{P_{0}}\right)^{\beta}
$$

with $S_{u 0}$ is the value of flame velocity at standard conditions: $P_{0}=1 \mathrm{~atm}$ and $T_{0}=298 \mathrm{~K}$. $\alpha$ et $\beta$ are two parameters characterizing the dependence of flame velocity on temperature and pressure.

\subsubsection{Conductive heat losses}

The conductive heat transfer is derived from wall surface temperature and is modelled in the transient regime by resolving the one-dimensional heat equation in spherical coordinates for all gas slices. The temperature of the last slice is determined by applying flux equality: At each time step, the conductive heat flux is equal to the convective heat flux transferred to the wall.

The unsteady model of heat diffusion is modelled by a nodal approach (see Fig. 1). For each gas slice of radius $r i[i=1, \mathrm{~N}]$, the thermal conductance, $G i$, is defined as follows:

$$
\begin{gathered}
G_{i}=\frac{4 \cdot \pi \cdot \lambda_{i}}{1 / r_{i-1}-1 / r_{i}}, i \epsilon[2, N-1] \\
G_{1}=\frac{4 \cdot \pi \cdot \lambda_{1}}{1 / \text { epsilon }-1 / r_{1}}, \text { epsilon }=10^{-4} \mathrm{~m} \\
G_{N}=h \cdot S_{\text {chamber }}
\end{gathered}
$$

Where, $\lambda_{i}$ is the thermal conductivity of fluid slices (w. $\left.m^{-1} \cdot K^{-1}\right), h$ is the wall heat transfer coefficient (w. $m^{-2} \cdot K^{-1}$ ) and $S_{\text {chamber }}$ is the chamber surface $\left(\mathrm{m}^{2}\right)$.

The unsteady resolution of heat equation is described by a time-explicit system: 


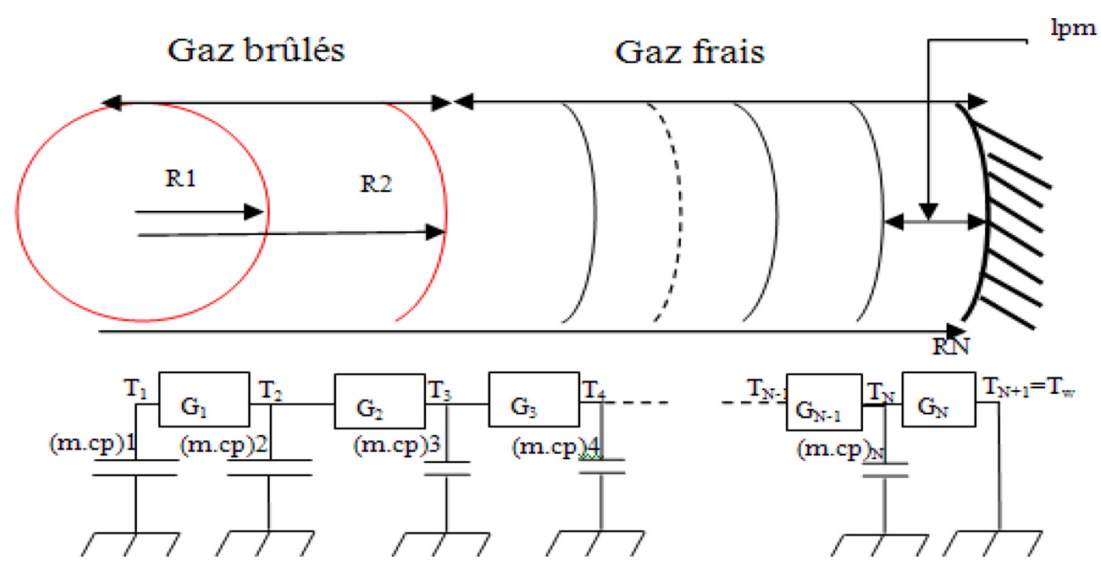

Figure 1: Heat conduction network in the vessel.

$$
\begin{gathered}
\left(m . C_{p}\right)_{1} \frac{T_{1, t+\Delta t}-T_{1, t}}{\Delta t}=G_{1}\left(T_{2, t}-T_{1, t}\right) \\
\left(m . C_{p}\right)_{i} \frac{T_{i, t+\Delta t}-T_{i, t}}{\Delta t}=G_{i}\left(T_{i+1, t}-T_{i, t}\right)+G_{i-1}\left(T_{i-1, t}-T_{i, t}\right), i \epsilon[2, N-1] \\
T_{N, t+\Delta t}=\frac{\left(T_{N-1, t+\Delta t}+\frac{G_{N} * T_{w}}{G_{N-1}}\right)}{\left(1+G_{N} / G_{N-1}\right)}, i=N
\end{gathered}
$$

For the numerical scheme, the stability criterion is taken into account by introducing the Freidrich-Lewy condition. The time step should assess the following inequality: $0 \leq \alpha \Delta t / \Delta r^{2} \leq \frac{1}{2} . \alpha$ is the thermal diffusivity $\left(\mathrm{m}^{2} . \mathrm{s}^{-1}\right)$.

\subsubsection{Radiative heat transfer}

The heat transferred to the wall through radiation comes from the hot, burnt gases. Radiative heat losses are simulated using the Stephan-Boltzmann law considering burnt gas as a grey body with a uniform temperature equal to the mean temperature of burnt gases, $T_{g b}$ and an emissivity equal to $\varepsilon$. The wall is considered as a black body with a constant temperature, $T_{w}$ and absorptivity $\alpha \simeq 1$. Only $\mathrm{CO}_{2}$ and $\mathrm{H}_{2} \mathrm{O}$ radiations are taken into account. For a given position of the flame front $r<R$ (radius of the chamber), the radiative heat flux received by the wall is expressed as follows:

$$
Q_{\text {wray }}(r)=\frac{4 \pi r^{2}}{4 \pi R^{2}} \alpha \cdot \varepsilon \cdot \sigma \cdot\left(T_{g b}^{4}-T_{w}^{4}\right)
$$

\subsubsection{Quenching distance}

The quenching distance is chosen as a simple criterion to stop the propagation of the flame approaching the wall. In the computation process, the quenching criterion is simulated by using the formulation mentioned in [5]: 


$$
\varphi=\frac{1}{P_{e}+1} \leftrightarrow P_{e}=\frac{1-\varphi}{\varphi}
$$

where $\varphi$ and $\mathrm{P}_{\mathrm{e}}$ were previously defined (see $\S .2 .1$ ).

\section{NUMERICAL RESULTS}

\subsection{Comparison between experimental and numerical results}

Numerical simulations are reported for the same experimental conditions mentioned previously (see \$3.1). Results of simulations are then, compared to experimental measurements to validate our approach and to prove the extended validity of this model to other thermal configurations such as the internal combustion engines.

Figure 2 shows the simulation for a stoichiometric methane-air mixture of initial pressure of $0,4 \mathrm{MPa}$ and initial temperature of $300 \mathrm{~K}$ in the steel vessel of $82 \mathrm{~mm}$ of diameter. Combustion stops according to the quenching criterion based on the Peclet number and where the quenching distance is less than $100 \mu \mathrm{m}$.

The evolution of wall heat flux is well simulated by the present model. The results show two major phases of heat transfer: for $t<t_{\text {pic }}=48 \mathrm{~ms}$ (the experimental time peak), the heat flux is low and does not exceed $0.4 \mathrm{MW} / \mathrm{m}^{2}$. This phase represents essentially the pressure rise effects on fresh gas temperature. As the flame propagates towards the wall, wall heat flux increases slightly to $2.2 \mathrm{MW} / \mathrm{m}^{2}$. The steep increase of heat flux is due to the flame quenching on the cold wall. Afterwards, heat transfer starts decreasing due to the gas cooling.

Overall, the combustion and the cooling phases are correctly simulated as regards heat flux. Nevertheless, a difference in the pressure during the peak of flame quenching is observed. During combustion, the quenching phenomenon happens because of the heat losses, essentially, which are large enough to slow down chemical reactions. In fact, all combustion power supposed to heat the preheated zone slightly goes to the wall by conduction through the fresh

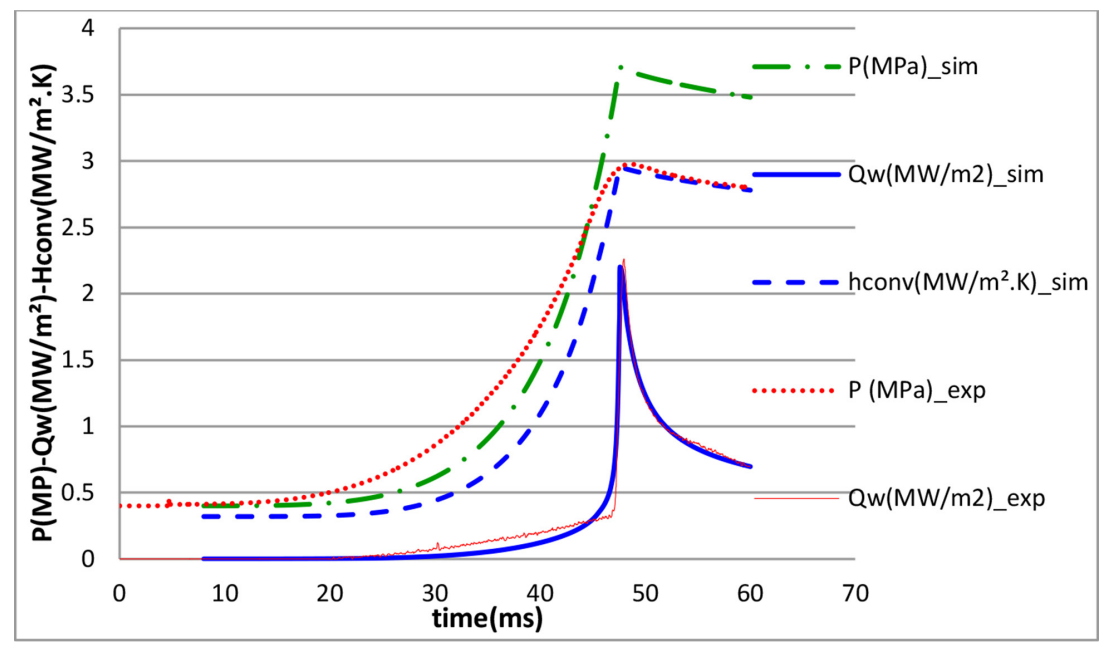

Figure 2: Comparison between experiments and simulations. 
gases. This is the large heat transfer, which takes place near the wall and which is finely described in this study.

After the heat losses peak, heat flux starts decreasing due to the cooling of hot gases. It is worth noting that we observe simultaneous maxima of wall heat flux and pressure reproduced by experiments and simulations. This feature enables to validate the spherical propagation of the flame whose modeling does not take into account of several disruptive phenomena such as the Archimedes' forces or the Darrieus-Landau instabilities.

One can see that comparison between experiments and simulations shows good agreements as regards heat flux and pressure appearances. Concerning heat flux, the simulations give satisfactory results and the heat flux peak computed is equal to the experimental value, which is equal to $2.2 \mathrm{MW} / \mathrm{m}^{2}$. Nevertheless, the pressure curve is not perfectly fitted comparing to the experimental results. A gap is observed when the flame approaches the wall and the simulated peak pressure is equal to 3.7 MPa comparing to the experimental value of $2.9 \mathrm{MPa}$. This difference can be due to the thermal expansion effects in fresh gases after diffusion, which widen by approaching the wall. This allows increasing the temperature in the majority of slices and the fact that pressure must follow the temperature evolution to conserve the constant volume of the chamber according to the perfect gas law.

\subsection{Conductive and radiative heat contributions}

Figure 3 shows the time evolution of the conductive and radiative heat transfers.

One can see that conductive and radiative heat fluxes increase in combustion phase then start decreasing in the cooling phase, simultaneously.

The conductive heat flux curve shows the same evolution of the total heat flux curve. In fact, a steep peak is observed at flame-wall interaction and is equal to $1.83 \mathrm{MW} / \mathrm{m}^{2}$ which represents $80 \%$ of the total heat flux at flame quenching. As far as heat radiation is concerned, it prevails conduction in combustion phase, especially before the flame quenching. It varies from $100 \%$ to $20 \%$ at quenching. During the cooling period, conduction remains greater than

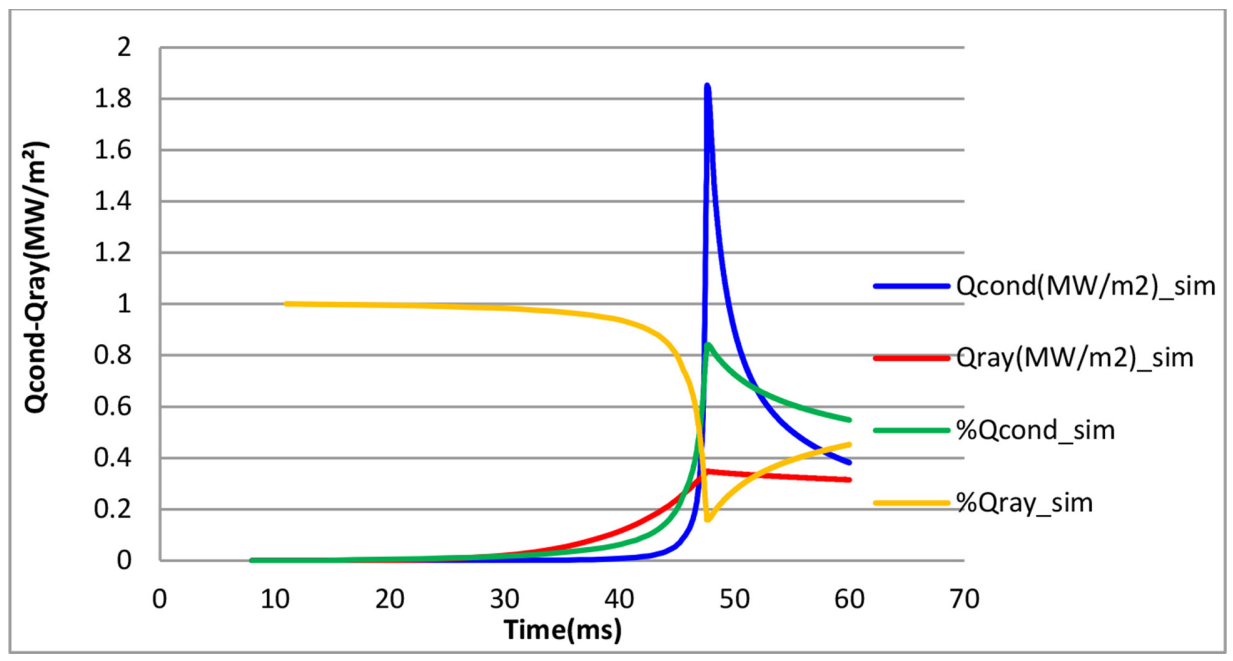

Figure 3: Comparison between conductive and radiative heat flux. 
radiation until the end of the cooling phase where conduction and radiation remain equal to $55 \%$ and $45 \%$, respectively.

\subsection{Temperature profile}

In our study, the time evolution of the temperature in the vessel, shown in Fig. 4, follows the heat flux and the pressure evolutions. According to Fergusson and Keck [6], the temperature profile in fresh gases is supposed to be linear. Simulations show that since the beginning of combustion, the fresh gas temperature seems to be linear and slightly increases during the whole quenching process. For different radius positions in the chamber, temperature evolution seems to be in accordance with the flame propagation. In fact, as the flame propagates through the wall, thermal expansion due to the increase of burnt gas slices causes the increase

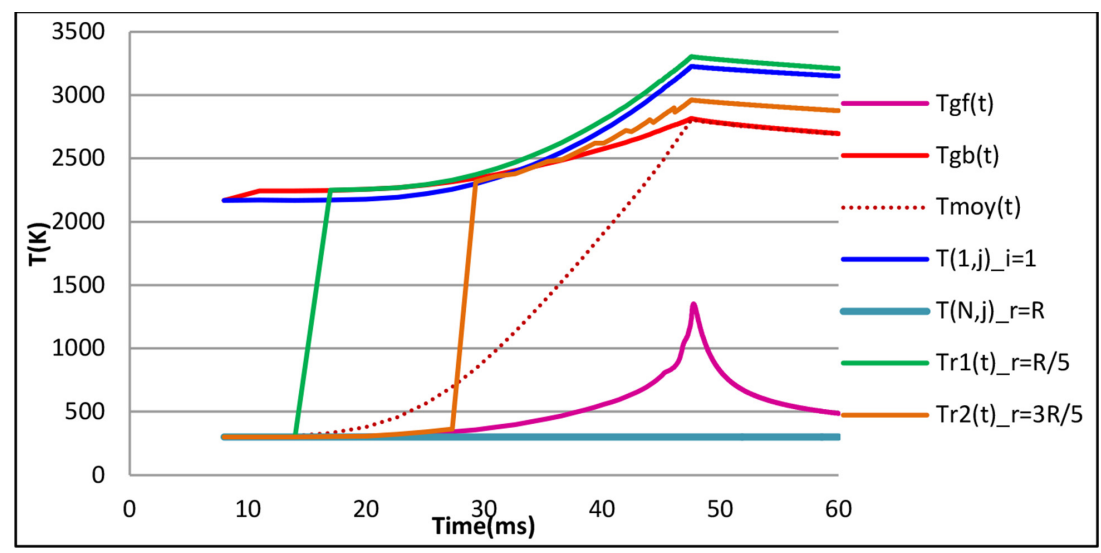

Figure 4: Temporal profiles of temperature in the combustion chamber.

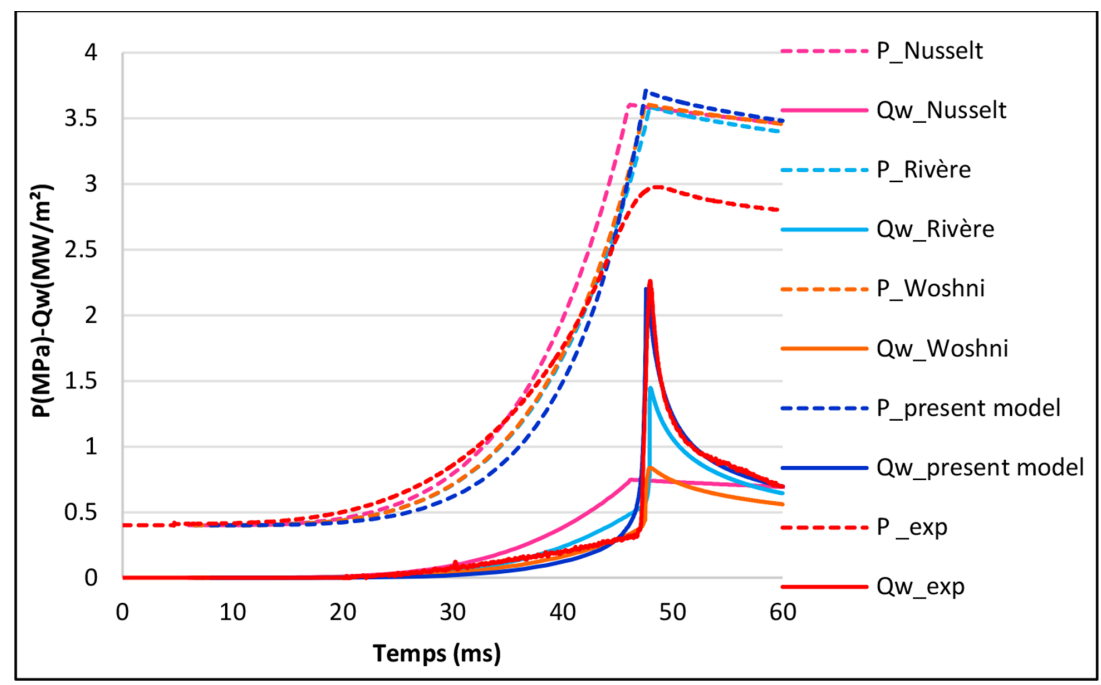

Figure 5: Comparison of the present model to the literature. 
of temperature during the combustion period. In addition, in Fig. 4 a higher temperature gradient is evident. The quenching temperature at the highest gradient of the heat release rate is $1350 \mathrm{~K}$ for the fresh gases and $2816 \mathrm{~K}$ for the burnt gases.

\subsection{Comparison of the literature models}

In this work, other existing models were implemented in the code to estimate the heat release. As we can see in Fig. 5, the evolution of wall heat flux is better simulated by the present model. In fact, the steep increase in heat flux is only simulated by models coming from an atomic-scale approach. Nevertheless, the Rivère model's [12] does not perfectly reproduce the heat flux peak and the instant of quenching. The heat losses computed by Woshni [10] are not suited to computing local unsteady heat losses as it does not take into account the reactive flow feature. The model stated by Nusselt [9] is also inadequate as it is based on a global approach.

\section{CONCLUSION}

In this work, a short review of wall heat losses in a spherical combustion vessel at constant volume has been presented. In the literature, no physical model is able to well reproduce the heat flux peak at the flame-wall interaction. The results of the present study show the ability of the atomic-scale model in rarefied regime to reproduce the behavior of the flame front during its interaction with the wall and give satisfactory results in the combustion and the cooling gas periods.

To sum up, the present model is well suited to correctly model the time-resolved and local heat losses. Nevertheless, it needs to take into account a fine spatial and temporal discretization to ensure a good numerical convergence.

\section{REFERENCES}

[1] Boust, B., Etude expérimentale et modélisation des pertes thermiques pariétales lors de l'interaction flamme-paroi instationnaire. Thèse de doctorat de l'université de Poitiers, 2006.

[2] Huang, W.M., Vosen, S.R. \& Greif, R., Heat transfer during laminar flame quenching: effect of fuels. Proceeding of the 21st Symposium on Combustion, pp. 1853-1860, 1986.

[3] Popp, P. \& Baum, M., Analysis of wall heat flux reaction mechanisms and unburnt hydrocarbons during the head-on quenching of a laminar methane flame. Combustion and Flame, 108, pp. 327-348, 1997. http://dx.doi.org/10.1016/S0010-2180(96)00144-7

[4] Potter, Jr., A.E. \& Berlad, A.L., A thermal equation for flame quenching, NASA TN 3398, 1955.

[5] Boust, B., Sotton, J., Labuda, S.A. \& Bellenoue, M., A thermal formulation for singlewall quenching of transient laminar flames. Combustion and Flame, 149, pp. 286-294, 2007. http://dx.doi.org/10.1016/j.combustflame.2006.12.019

[6] Fergusson, C.R. \& Keck, J.C., On laminar flame quenching and its applications to spark ignition engines. Combustion and Flame, 28, pp. 197-205, 1977. http://dx.doi.org/10.1016/0010-2180(77)90025-6

[7] Vosen, S.R., Greif, R. \& Westbrook, C.K., Unsteady heat transfer during laminar flame quenching, Proceeding of the 20th Symposium on Combustion, pp. 75-83, 1984. 
[8] Sotton, J., Boust, B., Labuda, S.A. \& Bellenoue, M., Head-on quenching of transient laminar flame: heat flux and quenching distance measurements. Combustion Science and Technology, 177, pp. 1305-1322, 2005. http://dx.doi.org/10.1080/00102200590950485

[9] Nusselt W., Der wärmeübergang in der Verbrennungskraftmaschine, V.D.I. Forschungsheft, p. 264, 1923.

[10] Woschni, G., A universally applicable equation for the instantaneous heat transfer coefficient in the internal combustion engine, SAE Technical Paper 670931, 1967.

[11] Han, Z. \& Reitz, R.D., A temperature wall function formulation for variable-density turbulent flows with application to engine heat transfer modeling. International Journal of Heat and Mass Transfer, 40, pp. 613-625, 1997. http://dx.doi.org/10.1016/0017-9310(96)00117-2

[12] Rivère, J.P. \& Mechkor, M., Modélisation des échanges thermiques sur la paroi de la chambre de combustion, Rapport RENAULT, 2005.

[13] Carminati, R., Transport en milieux dilués (chapter 2). Micro et nanothermique, ed S. Volz, Editions du CNRS: Paris, France, pp. 34-35, 2007.

[14] Metghalchi, M. \& Keck, J.C., Laminar burning velocity of propane-air mixtures at high temperature and pressure. Combustion and Flame, 48, pp. 191-210, 1980.

http://dx.doi.org/10.1016/0010-2180(82)90127-4 